\title{
Dynamic Gene Expression Profile Changes in Synovial Fluid Following Meniscal Injury; Osteoarthritis (OA) Markers Found
}

\author{
Danica D. Vance ${ }^{1 *}$, Liyong Wang², Evadnie Rampersaud ${ }^{2}$, Bryson P. Lesniak ${ }^{1}$, Jeffery M. Vance ${ }^{2}$, \\ Margaret A. Pericak-Vance ${ }^{2}$ and Lee D. Kaplan ${ }^{1}$ \\ ${ }^{1}$ UHealth Sports Performance and Wellness Institute, University of Miami, Miller School of Medicine, Miami, Florida \\ ${ }^{2}$ John P. Hussman Institute for Human Genomics, University of Miami, Miami Miller School of Medicine, Miami, Florid
}

Received: 02 June, 2014; Accepted: 04 July, 2014; Published: 18 July, 2014

*Corresponding author: Danica D. Vance, UHealth Sports Performance and Wellness Institute, University of Miami, Miller School of Medicine, Miami, Florida, USA, 33136,Tel: 305-781-1766; E-mail:ddv305@gmail.com

\begin{abstract}
Purpose: We hypothesize that molecular changes leading to Osteoarthritis $(\mathrm{OA})$ occur soon after a meniscal tear and long before the manifestation of $\mathrm{OA}$. We sought to characterize the gene expression profile in synovial fluid at different time points following a meniscal tear with a focused examination on inflammatory and arthritis related genetic markers.
\end{abstract}

Methods: Synovial fluid was collected from 8 male patients (24 48yrs old) with evidence of meniscal injury on Magnetic Resonance Imaging (MRI). Illumina humanht-12 microarray and RNA-Seq were used to characterize gene expression profile in cell pellets and supernatant, respectively. Unsupervised clustering analysis was performed to identify patterns of gene expression profile among samples. Metacore was utilized to perform pathway analysis. T-tests with False Discovery Rate (FDR) corrections were used to compare subset of genes among individuals.

Results: Individuals with short injury duration (<2 months) had distinct expression signatures than the rest, in both cell pellet and supernatant analyses. In the cell pellets, among genes previously linked to inflammatory or arthritic conditions, IL 1B, IL1RN, ILF2, NFKB1, IL10RB, IL18BP, ILF3, IL13RA, BMP2K and IL10RB were significantly upregulated (adjusted $\mathrm{p}<0.05$ ) in individuals with long duration compared to individuals with shorter injury duration in the microarray analysis. In the cell-free supernatant, 764 RNA species were identified and 65 RNA species were down-regulated and 78 RNA species were up-regulated (FDR $<0.05$ ) in individuals with short compared to individuals with long injury duration. The most differentially expressed gene in the supernatant is SLC2A9 (P = $1.2 \times 10_{-13} ; \mathrm{FDR}=9.6 \times 10_{-11}$ ). In the cell pellet, the top 5\% expressed genes are enriched for inflammatory and cytoskeleton remodeling pathways. Among them LAIR 1, TMSB4X, CCR6, IL18 and IL10; all have been implicated in arthritic conditions.

Conclusions: Molecular changes contributing to OA development occur earlier than previously described and potentially evolve or change over time.

Clinical Relevance: Our data suggest the potential for early interventions to halt detrimental molecular changes following meniscal injury, thereby reducing the susceptibility of patients to $\mathrm{OA}$ following a meniscal tear.

Keywords: Meniscus; Meniscal tear; Gene expression; Synovial fluid; Microarray; Osteoarthritis

\section{Introduction}

The meniscus is a critical component of the knee joint, contributing to shock absorption, load distribution and joint stability [1]. A meniscal tear is one of the most common injuries seen in orthopedics [2]. In addition to the physical symptoms and limitations caused by the acute injury, researchers have linked meniscal injury to the progression and development of osteoarthritis $(\mathrm{OA})[1,3,4]$. Following meniscal damage, changes in load distribution contribute to joint space narrowing and increased mechanical stresses on articular cartilage, which in turn lead development of OA [1]. Osteoarthritic changes in articular cartilage have been seen as early as 2 years postmeniscal damage [4].

In addition to the mechanical changes occurring in the knee joint following meniscal tears, differences at the molecular level in both meniscal tissue and synovium have been reported [57]. RNA extracted from injured meniscal tissue was found to have significantly elevated levels of several arthritis-related genetic markers in patients under the age of 40 [5]. Similarly, Scanzello et al. [6] also found significant differences in gene expression patterns in synovium tissue after a meniscal injury when comparing patients with evidence of inflammation to those without inflammation [6,7].

Yet to be extensively investigated are the dynamic molecular changes that occur in the synovial fluid following a meniscal tear. Because the meniscus tissue has limited blood supply, the synovial fluid is an important source of nutrients and waste products. Previous studies have successfully used synovial fluid to examine protein complexes and specific cytokines that might contribute to acute knee pain [8-10]. This is one of the first studies to look at gene expression globally in synovial fluid following a meniscal tear, one of the major risks for development of OA in the knee. Indeed, radiographic OA changes in cartilage can be seen as early as 2 years post-injury. However, changes at the molecular level could occur earlier. Examining gene expression of synovial fluid following a meniscal tear will provide further insights on the inflammatory and cellular changes that occur in the knee joint 
after injury. Determining these changes and when they begin would be critical to successful intervention following meniscal injury to prevent or delay the development of OA. Furthermore, if a link can be established between these molecular changes and clinical outcomes including future degenerative changes in the knee joint, these data could potentially provide a non-invasive method to help identify those individuals who are at high risk of developing OA. Early stage identification of OA patients makes room for potential therapeutic interventions when the interventions are probably the most effective. We hypothesize that molecular changes leading to OA occur soon after a meniscal tear and long before the manifestation of OA. Our purpose was to characterize the gene expression profile of synovial fluid following a meniscal tear to determine if known $\mathrm{OA}$ and inflammatory genes can be detected early post-injury.

\section{Methods}

\section{Synovial fluid collection}

The Institutional Review Board (IRB) (Protocol \#: 20100506) of the participating institution approved this study. Patients were enrolled through the sports medicine clinic. Potential study participants were individuals scheduled to undergo a partial arthroscopic meniscectomy (Figure 1). Subjects were less than 50 years of age, had no previous knee pathology or surgery of the injured knee including any ligamentous or cartilage injury, and had MRI evidence of a meniscal tear. Additional exclusion criteria included patients with Kellgren- Lawrence Grade III or Grade IV osteoarthritis of the knees observed during the operation, Body Mass Index (BMI) greater then 30, age $>50$ years old or those diagnosed with one of the following; systemic rheumatoid arthritis, neuromuscular disease or diabetes. These exclusion criteria were met to limit the presence of osteoarthritis within the knee joint. Synovial fluid from the affected knee joint was collected by needle aspiration from eight male patients $(24 \sim 48$ years old) at the time of surgery, prior to initial incision. Collected fluid was immediately transferred to RNAse free $1.5 \mathrm{ml}$ endorphin tubes and kept on ice while transferring to the lab for processing. In addition, medical information regarding injury phenotype was collected directly from patient and the patient's clinic notes (Table 1).

\section{Synovial Fluid processing}

All sample processing and molecular analysis were carried out at Hussman Institute for Human Genomics (HIHG), University of miami. In preparation for RNA extraction, collected synovial fluid samples were centrifuge at $1500 \mathrm{~g}$ at $4^{\circ} \mathrm{C}$ for 5 minutes or until pellet formation. Next, the supernatant was removed and $1 \mathrm{ml}$ of qiazol lysis reagent was added to the pellet followed by vigorous vortexing. Supernatant and cell pellet were stored at $-80^{\circ} \mathrm{C}$ until RNA extraction.

\section{Rna extraction}

Total RNA was extracted from cell pellet using the qiazol-chloroform-isopropanol protocol as instructed by the manufacture (Qiagen). Cell-free RNA was extracted using rneasy kit (Qiagen) with modified protocol to recover small RNA species. RNA was quantified by Qubit $®$ RNA assay (Invitrogen Life Technologies) and qualified by Agilent 2100 RNA 6000 Pico Chip (Agilent Technologies).

\section{Microarray and RNA-Seq Analysis}

For the cell-pellet RNA, genome-wide gene expression profiling was done using the Illumina Whole Genome High Throughput (HT) 12 Assay. For the cell-free RNA, nebnext $\AA$ Small RNA Library Prep Set for Illumina ${ }^{\circledR}$ was used for library preparation. Next-generation sequencing of these libraries were carried out on the Illumina hiseq 2500 instrument. Both microarray and RNA-Seq were performed at Center for Genomic Technology at the Hussman Institute for Human Genomics following manufacturer's instructions.

\section{Statistical data analysis}

All statistical analyses were performed in the Center of Genetic Epidemiology and Statistical Genetics at the HIHG. For the microarray data, the raw expression data ( $\log _{2}$ values) were transformed using variance-stabilizing transformation (VST) [11] with the LUMI Bioconductor R package [12], which takes into account the large number of technical replicates on
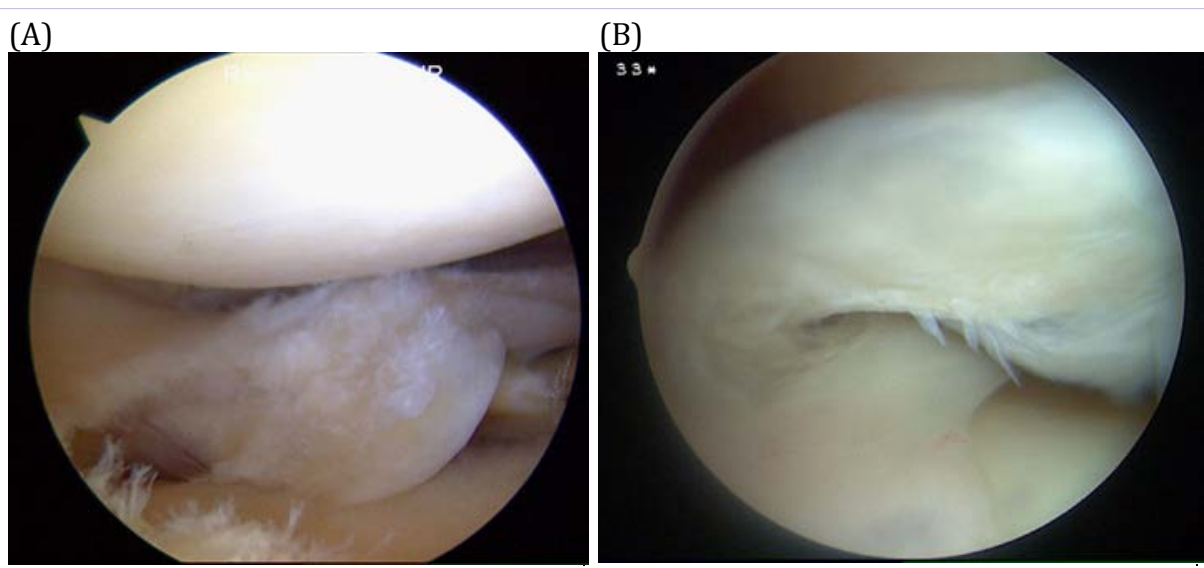

Figure 1: Arthroscopic views of meniscal tear. (A) Complex degenerative tear (B) Flipped bucket handle tear. 
Table 1: Data demographics.

\begin{tabular}{|c|c|c|c|c|c|c|c|}
\hline Samples & Age & $\begin{array}{c}\text { Traumatic } \\
\text { injury }\end{array}$ & $\begin{array}{c}\text { Injury } \\
\text { Duration }\end{array}$ & $\begin{array}{c}\text { Injury Location } \\
\text { (LM= Lateral } \\
\text { Meniscus, } \\
\text { MM=Medial } \\
\text { Meniscus) }\end{array}$ & Effusion & $\begin{array}{c}\text { Type of Meniscal } \\
\text { Tear }\end{array}$ & $\begin{array}{c}\text { OA Grades (Kellgren and } \\
\text { Lawrence ) (P=Patella, } \\
\text { T=Trochlear, MFC=medial } \\
\text { femoral condyle, LFC=Lateral } \\
\text { femoral Condyle) }\end{array}$ \\
\hline $\mathbf{1}$ & 24 & yes & 4 months & Left LM & mild & Parrot-Beak & P=Grade 1 lesion \\
\hline $\mathbf{2}$ & 48 & No & 4 months & Left LM & moderate & Discoid & no changes observed \\
\hline $\mathbf{3}$ & 28 & no & 7 months & Right MM & none & $\begin{array}{c}\text { Complex, } \\
\text { Degenerative }\end{array}$ & P=Grade 2 lesion \\
\hline $\mathbf{4}$ & 38 & no & 3 months & Right MM, LM & none & $\begin{array}{c}\text { MM: Horizontal, } \\
\text { LM=radial }\end{array}$ & MFC=Grade 1-2 lesion \\
\hline $\mathbf{5}$ & 39 & yes & 2 months & Right MM & mild & $\begin{array}{c}\text { Double Flap of } \\
\text { Posterior Horn }\end{array}$ & $\begin{array}{c}\text { P =Grade 2 lesion, T=Grade 2 } \\
\text { lesion }\end{array}$ \\
\hline $\mathbf{6}$ & 41 & yes & 1 month & Right MM & mild & $\begin{array}{c}\text { Complex, } \\
\text { Degenerative of } \\
\text { posterior horn }\end{array}$ & $\begin{array}{c}\mathrm{P}=\text { Grade 2 lesion, MFC=Grade } \\
2 \text { lesion }\end{array}$ \\
\hline $\mathbf{7}$ & 30 & no & 1 month & Left MM & mild & $\begin{array}{c}\text { Flipped bucket } \\
\text { handle }\end{array}$ & P =Grade 1 changes \\
\hline $\mathbf{8}$ & 44 & yes & 2 months & Left MM & mild & $\begin{array}{c}\text { Complex, } \\
\text { Degenerative }\end{array}$ \\
\hline
\end{tabular}

the Illumina arrays. Normalization was conducted using the Robust Spline Normalization (RSN) algorithm, which combines features of quantile and loess normalization. Quality control was performed using the lumiQ command. For the RNA-Seq data, the STAR program was used to align the trimmed sequencing reads against human reference genome to localize RNA species. Given the wide range of number of reads generated for each sample, normalization is an essential step. The EdgeR program normalizes the reads by estimating effective library size based on the depth of reads. The normalized gene expression unit is Count Per Million (CPM).

In order to correlate the RNA expression profile with clinical characteristics, unsupervised cluster analysis was conducted using LUMI software for microarray data and the EdgeR program for RNA-Seq data. For gene expression analysis, we first characterized what genes are expressed in synovial fluid following meniscal injury, examining those genes with the greatest average expression (top 5\%) across all 8 samples. Pathway analysis was conducted using MetaCore ${ }^{\mathrm{TM}}$ (GeneGo, Inc.) software. Then, we carried out differential gene expression analysis comparing samples with short duration time ( $\leq 2$ months) to samples with longer duration time ( $\geq 3$ months). For the microarray data, T-tests with FDR corrections [13] were performed to compare the gene expression profiles of a subset of OA-related genes (n = 203 genes, 340 transcripts) within samples of varied injury duration. Genes with $p$ value $<0.05$ were considered significant For the RNA-Seq data, EdgeR was used to evaluate differential gene expression among the 764 RNA species identified in the cellfree synovial fluid.

\section{Results}

For this pilot study, we were able to ascertain eight male patients with available funds. Average age of study participant was $36.5 \pm 8.3$ years old (range; $24-48$ yrs). For all patients, initial assessment of injury was made in clinic and then confirmed by MRI by an independent musculoskeletal radiologist. All 8 patients underwent an arthroscopic partial meniscectomy for a meniscal tear (Figure 1). The type of meniscal tear, degree and location of arthosis were systematically observed by the surgeon during the operation (Table 1). Additional injury phenotype was collected from the patient and initial clinic note (Table 1). Specifically, duration of injury was calculated by the self-reported initial injury time and the time of sample collection, i.e. time of surgery. The median value for time between initial injury and surgery was 4 months (range: 1 month to 7 months).

The top 5\% expressed genes in cell pellets across all samples are enriched for pathways involved in oxidative phosphorylation, immune response and cytoskeleton remodeling. (Supplementary Figure 1) Included in the top 5\% expressed genes were several genes with previously reported association to inflammation and/ or Rheumatic or Osteoarthritis; such as LAIR 1, TMSB4X, CCR6, IL18 and IL10 [2, 14-17].

Unsupervised cluster analysis of gene expression profile is illustrated in Figure 2 (heat map of microarray data in cell pellet) and Figure 3 (principal component analysis of RNA-Seq data in cell-free supernatant). With the gene expression profile in cell pellets, we observed a clustering of three samples (samples 6 8) with distinct gene expression profiles from the rest (Figure 2). When we compared different injury phenotypes including; age, etiology of injury, injury duration, location of injury (medial vs. Lateral meniscus) and presence of effusion (Table 1), we found that injury duration best stratified the expression patterns. Specifically, samples 6,7 , and 8 had the shortest injury duration ( $\leq 2$ months). Sample 5 also had a 2 months of injury duration and was clustered right next to the tight cluster formed by samples 6 8. With the gene expression profile in cell-free supernatant, we observed a clustering of samples $6 \sim 8$, as seen in the microarray analysis. Samples with the longest injury duration 


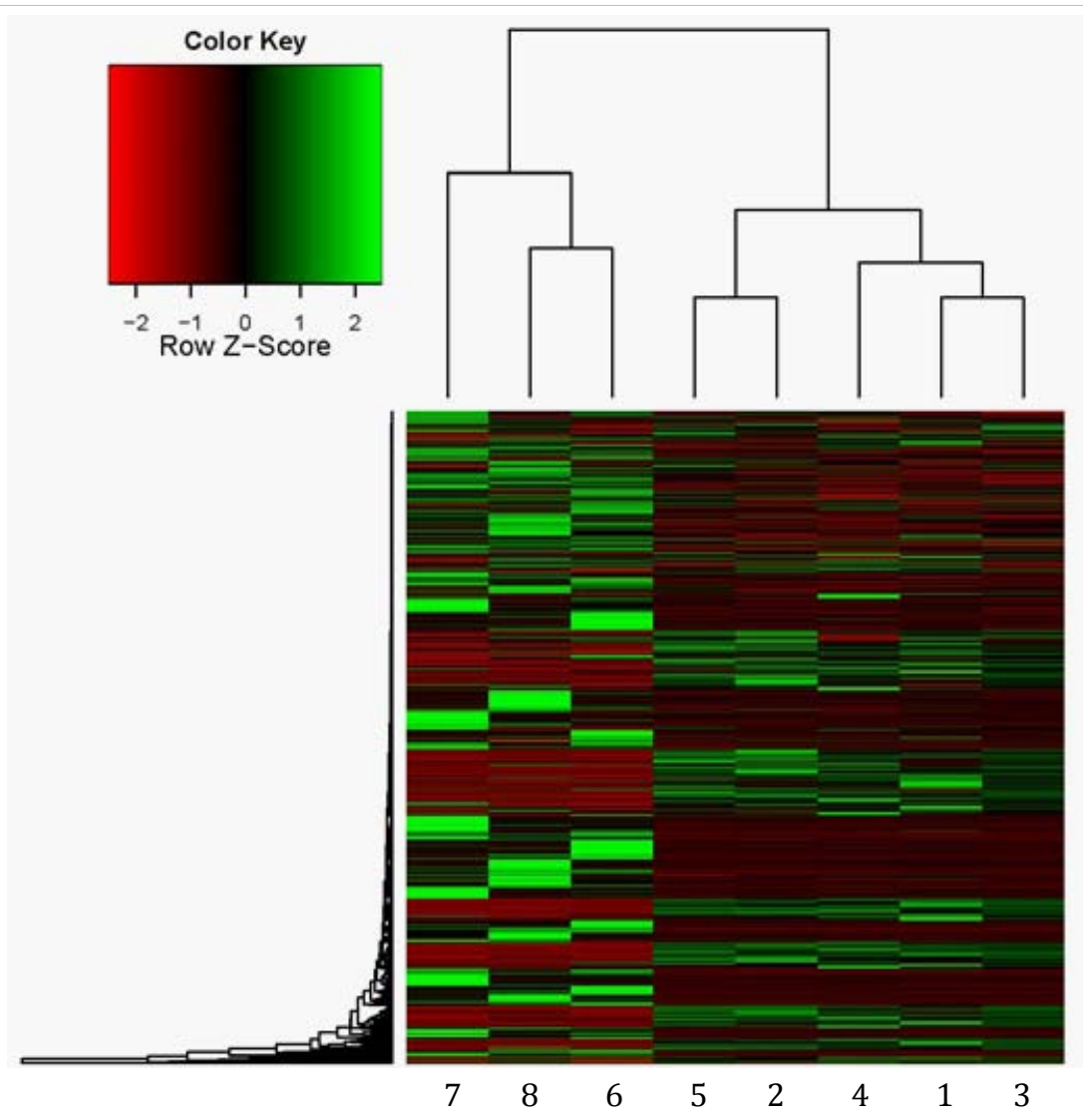

Figure 2: Unsupervised cluster analysis of gene expression in cell pellet.

Heatmap showing expressed genes across individuals. Horizontal axis displays individual samples, vertical axis displays each expressed genes by z-scores (scaled value of normalized intensity scores). Red=decreased intensity; green=increased intensity.

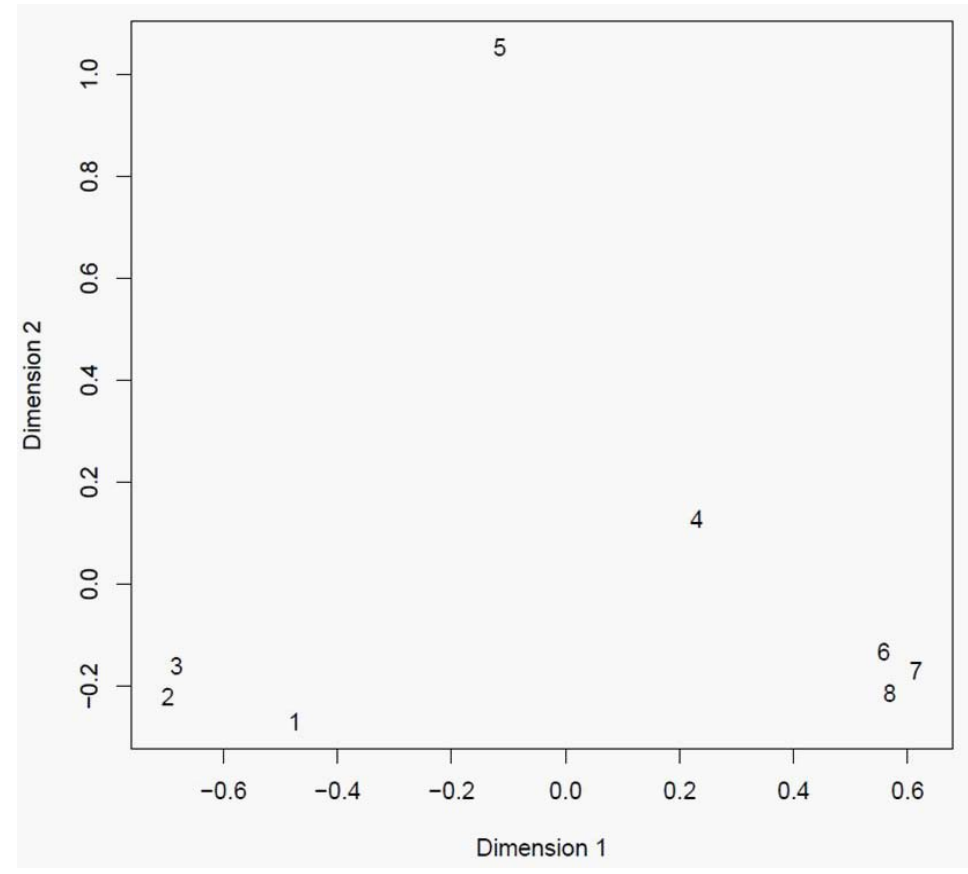

Figure 3: Unsupervised cluster analysis of gene expression in cell-free supernatant. 
( $\geq 4$ months), i.e. Sample 1 3, were clustered on the other end of first dimension (Figure 3).

To examine the gene expression differences between samples with short versus long injury duration we examined the fold change in a subset of 203 genes (340 transcripts) with reported involvement in either inflammatory or osteoarthritis pathways $[13,18-27]$, that were expressed in the cell pellet of synovial fluid. The complete list of genes can be found in (supplementary Table 1). The following genes met FDR Corrected significance ( $\mathrm{p}$ $<0.05$ ) (Table 2): Interleukin-1 beta (IL1B), Interleukin 1 receptor antagonist (IL1RN), Interleukin enhancing binding factor 2 (ILF2), Nuclear Factor of Kappa light polypeptide gene enhancer in B-cells 1(NFKB1), Interleukin 10 receptor beta (IL10RB), Interleukin 10 receptor alpha (IL10RA) Interleukin 18 binding protein (IL18), Interleukin enhancing binding factor 3 (ILF3), Interleukin 13 receptor, alpha 1 (IL13RA), Bone morphogenetic protein 2 inducible kinase (BMP2K).

In the cell-free supernatant, 764 RNA species were identified to be present and all of them were evaluated for differential expression between samples with shorter ( $\leq 2$ months) and longer ( $\geq 3$ months) injury duration. 65 RNA species were downregulated and 78 RNA species were up-regulated (FDR $<0.05$ ) in individuals with short injury duration. The most differentially expressed gene in the supernatant is $\operatorname{SLC} 2 A 9\left(\mathrm{P}=1.2 \times 10^{-13}\right.$; FDR $\left.=9.6 \times 10^{-11}\right)($ Table 3$)$.

\section{Discussion}

While examining, highly expressed genes in the synovial fluid from patients with meniscal injury having no OA symptoms, we observed that the expressions of many genes, due to the early injury are shown to be associated with $\mathrm{OA}$ and inflammation. These reported genes include LAIR1, TMSB4X, CCR6, IL18, IL10 $[2,14-17]$. LAIRI is the highest expressed gene among all the samples. LAIRI is an immune inhibitory receptor for collagen found on the most immune cells (pubmed gene ID: 3903) [28]. Its inhibitor LAIR2 is found in the synovial fluid of patients having Rheumatoid Arthritis (RA) or OA. Patients with RA show higher expression level of LAIR2. Similarly, IL-10, a prominent chondroprotective anti-inflammatory marker released by the synovium and cartilage, was also highly expressed across all samples. IL-10 has been shown to be present in synovial fluid of OA patients [29].

The second highest average expressed gene across all samples was TMSB4X, which encodes a protein (TB4) that activates the expression of MMPS in fibroblasts (pubmed ID: 7114). Recently, TB4 level in synovial fluid of patients with RA was found when compared to OA patients. It was also significantly associated with the levels of $M M P-9, M M P-13, I L-6$ and $I L-8$ [15], suggesting TB4 may play an early role in starting the inflammatory and cartilage degradation cascade.

Table 2: Differentially expressed OA-related genes in cell pellets between samples with short and long injury duration.

\begin{tabular}{|c|c|c|c|}
\hline Gene Symbol & log FC & Average Expression (Z scores) & P Value \\
\hline IL1RN & -1.36 & 7.31 & 0.0007 \\
\hline IL13RA1 & -2.67 & 8.46 & 0.024 \\
\hline IL10RB & -1.53 & 7.55 & 0.024 \\
\hline IL10RA & -2.04 & 8.05 & 0.0015 \\
\hline ILF3 & -1.36 & 7.44 & 0.0016 \\
\hline ILF2 & -2.19 & 8.15 & 0.0019 \\
\hline IL1B & -1.45 & 7.36 & 0.0021 \\
\hline IL18BP & -1.53 & 7.51 & 0.024 \\
\hline BMP2K & -1.09 & 7.47 & 0.024 \\
\hline NFKB1 & -1.88 & 9.15 & 0.024 \\
\hline
\end{tabular}

Table 3: Top differentially expressed RNAs in supernatant between samples with short and long injury duration.

\begin{tabular}{|c|c|c|c|c|c|}
\hline Gene Symble & Type of RNA & $\log \mathrm{FC}$ & Average Expression (logCPM) & P Value & FDR \\
\hline SLC2A9 & protein_coding & -7.37 & 1.95 & $1.21 \mathrm{E}-13$ & $9.60 \mathrm{E}-11$ \\
\hline NBPF1 & lincRNA & -10.71 & -0.56 & $5.25 \mathrm{E}-13$ & $2.09 \mathrm{E}-10$ \\
\hline RP11-300A12.1 & pseudogene & -6.23 & 5.13 & $2.42 \mathrm{E}-12$ & $6.43 \mathrm{E}-10$ \\
\hline PCK2 & protein_coding & 11.78 & 6.83 & $4.17 \mathrm{E}-12$ & $8.32 \mathrm{E}-10$ \\
\hline FBX045 & protein_coding & -6.16 & 2.69 & $1.37 \mathrm{E}-11$ & $2.18 \mathrm{E}-09$ \\
\hline C1orf114 & protein_coding & -6.50 & 0.70 & $2.93 \mathrm{E}-11$ & 3.90E-09 \\
\hline N4BP2 & protein_coding & -7.20 & -0.31 & $4.82 \mathrm{E}-11$ & $5.48 \mathrm{E}-09$ \\
\hline MBTPS1 & protein_coding & -5.98 & 0.55 & $1.46 \mathrm{E}-10$ & $1.45 \mathrm{E}-08$ \\
\hline INHBB & protein_coding & -6.72 & -0.81 & $3.65 \mathrm{E}-10$ & $3.23 \mathrm{E}-08$ \\
\hline AL590452.1 & protein_coding & -5.96 & 1.64 & $4.15 \mathrm{E}-10$ & $3.30 \mathrm{E}-08$ \\
\hline
\end{tabular}


Additionally, CCR6 is a chemokine receptor that is selectively expressed on, and required for the migration of Interleukin 17-producing helper cells (TH17). TH17 cells have previously been shown to be involved in damaging immune processes within the knee joint $[16,17]$.

When overall gene expression patterns were compared using unsupervised clustering analysis, distinct patterns of expression were observed between individuals with short and long injury duration: which was seen in both the microarray analysis of RNA from cell pellets and RNA-Seq analysis of RNA from cellfree supernatant. In the cell pellet analysis, several genes with previous association to either inflammatory or OA processes were significantly differently expressed $(p<0.05)$ between the early and later samples (Table 2). Both IL1B and IL18BP members of the interleukin 1 cytokine family showed significantly increased expression in the longer duration samples. The Interleukin 1 family are proinflammatory cytokines that play key roles in initiating cartilage degradation pathways within joints $[8,18,30,31]$. Perhaps this increased expression is indicative of $I L-1$ cytokines proposed early role in joint damage.

Additionally, NFKB1 and BMP2K showed significantly higher expression in samples with longer injury duration. It plays a role in inflammation and synovium hyperplasia within the arthritic joint $[26,27]$. BMPK2 is a kinase induced by BMP-2 and plays important regulatory roles in attenuating BMP-2's biological actions. With roles in cartilage and bone formation, BMP-2 has been shown to be elevated in OA cartilage compared to controls, increasing proteoglycan synthesis and MMP degradation of aggrecan $[26,27]$. These variations in gene expression patterns seen within our data set raise the important question of whether the osteoarthritic processes at the molecular level begin to form soon after injury and evolve and change with time, creating susceptibility for later $\mathrm{OA}$ formation.

In the cell-free supernatant analysis, the most differentially expressed gene is $S L C 2 A 9$, which encodes a member of the solute carrier family 2, also known as facilitated glucose transporter, which plays a significant role in maintaining glucose homeostasis. SLC2A9 is expressed in normal articular chondrocytes and is induced by proinflammotry cytokines, such as IL-1beta \{PMID:11739520\}. It is likely that SLC2A9 may play a role in the development and survival of chondrocytes in cartilage matrices \{PMID:18841755\}.

This study has several limitations. Although efforts were made to standardize the procedure, synovial fluid collection by needle aspiration can be a variable clinical technique [10]. Some aspirates contained more blood then others, and the total amount of synovial fluid collected per patient also varied. However, no correlation with results was found with sample hemoglobin levels. In addition there could be other general health factors or injury characteristics (type of meniscus tear) not accounted for that could attribute to gene expression changes in synovial fluid. Although the goal of this study was to demonstrate the initial characterization of synovial fluid following a meniscal tear, we must take in account that there are no controls or uninjured synovial fluid samples to compare to. Our current data supports the need for a larger more extensive study where controls are also ascertained. Also, as a retrospective study, the duration of injury data collection was based on patient self-report, which has obvious limitations. Most importantly, the sample size $(n=8)$ limits the power of the study for a genome-wide non-biased analysis. Despite its small size, we were able to observe significant dynamic changes of some major OA gene findings in these data.

The results observed in this study are intriguing and lead the way for a larger longitudinal study looking at the gene expression changes occurring in synovial fluid following a meniscal tear. Indeed, Vidal et al. [10] has previously stated that the technique of identifying biomarkers in synovial fluid as "extremely valuable to the orthopedic community." Indeed once fully investigated, this minimally invasive method of synovial fluid analysis has the ability to aid clinicians in improving patient care in a variety of ways; whether it be used for early intervention or for predicting patient outcomes.

In conclusion, the study demonstrates that RNA can be easily obtained from synovial fluid following a meniscal tear and demonstrates the early expression in synovial fluid following injury of several previously associated OA genes. If true, this opens the possibility of a non-invasive method for early detection of those individuals following meniscal injury that are predisposed to developing OA. In addition, characterization of these dynamic molecular changes could provide novel insights on the process and mechanism of OA development.

\section{References}

1. Dong $\mathrm{Y}, \mathrm{Hu} \mathrm{G}$, Dong $\mathrm{Y}, \mathrm{Hu} \mathrm{Y}, \mathrm{Xu} \mathrm{Q}$. The effect of meniscal tears and resultant partial meniscectomies on the knee contact stresses: a finite element analysis. Comput Methods Biomech Biomed Engin. 2013; 17(13):1452-1463. doi: 10.1080/10255842.2012.753063.

2. Cattano NM, Driban JB, Balasubramanian E, Barbe MF, Amin M, Sitler MR. Biochemical comparison of osteoarthritic knees with and without effusion. BMC Musculoskelet Disord. 2011; 12:273. doi:10.1186/14712474-12-273.

3. Englund M, Guermazi A, Roemer FW, Aliabadi P, Yang M, Lewis CE, et al. Meniscal tear in knees without surgery and the development of radiographic osteoarthritis among middle-aged and elderly persons: The Multicenter Osteoarthritis Study. Arthritis Rheum. 2009; 60(3):831-839. doi: 10.1002/art.24383.

4. Chang A, Moisio K, Chmiel JS, Eckstein F, Guermazi A, Almagor O, et al. Subregional effects of meniscal tears on cartilage loss over 2 years in knee osteoarthritis. Ann Rheum Dis. 2011; 70(1):74-79. doi: 10.1136/ ard.2010.130278

5. Brophy RH, Rai MF, Zhang Z, Torgomyan A, Sandell LJ. Molecular analysis of age and sex-related gene expression in meniscal tears with and without a concomitant anterior cruciate ligament tear. J Bone Joint Surg Am. 2012; 94(5):385-393. doi: 10.2106/JBJS.K.00919.

6. Scanzello CR, McKeon B, Swaim BH, DiCarlo E, Asomugha EU, Kanda $\mathrm{V}$, et al. Synovial inflammation in patients undergoing arthroscopic meniscectomy: molecular characterization and relationship to symptoms. Arthritis Rheum. 2011; 63(2):391-400. doi: 10.1002/ art.30137.

7. Scanzello CR, Umoh E, Pessler F, Diaz-Torne C, Miles T, Dicarlo E, et 
al. Local cytokine profiles in knee osteoarthritis: elevated synovial fluid interleukin-15 differentiates early from end-stage disease. Osteoarthritis Cartilage. 2009; 17(8):1040-1048. doi: 10.1016/j. joca.2009.

8. Cuellar JM, Scuderi GJ, Cuellar VG, Golish SR, Yeomans DC. Diagnostic utility of cytokine biomarkers in the evaluation of acute knee pain. J Bone Joint Surg Am. 2009; 91(10):2313-2320. doi: 10.2106/ JBJS.H.00835.

9. Scuderi GJ, Woolf N, Dent K, Golish SR, Cuellar JM, Cuellar VG, et al. Identification of a complex between fibronectin and aggrecan G3 domain in synovial fluid of patients with painful meniscal pathology. Clin Biochem. 2010; 43(10-11):808-814. doi: 10.1016/j. clinbiochem.2010.04.069.

10. Scuderi GJ, Golish SR, Cook FF, Cuellar JM, Bowser RP, Hanna LS, et al. Identification of a novel fibronectin-aggrecan complex in the synovial fluid of knees with painful meniscal injury. J Bone Joint Surg Am. 2011; 93(4):336-340. doi: 10.2106/JBJS.J.00718.

11. Lin SM, Du P, Huber W, Kibbe WA. Model-based variance-stabilizing transformation for Illumina microarray data. Nucleic Acids Res. 2008; 36(2):11. doi: 10.1093/nar/gkm1075.

12. Du P, Kibbe WA, Lin SM. Lumi: a pipeline for processing Illumina microarray. Bioinformatics. 2008; 24(13):1547-1548. doi: 10.1093/ bioinformatics/btn224.

13. Benjamini Yoav, Hochberg Yosef. Controlling the false discovery rate: a practical and powerful approach to multiple testing. Journal of the Royal Statistical Society. 1995; 57(1):289-300.

14. Olde Nordkamp MJ, van Roon JA, Douwes M, de Ruiter T, Urbanus RT, Meyaard L. Enhanced secretion of leukocyte-associated immunoglobulin-like receptor 2 (LAIR-2) and soluble LAIR-1 in rheumatoid arthritis: LAIR-2 is a more efficient antagonist of the LAIR-1-collagen inhibitory interaction than is soluble LAIR-1. Arthritis Rheum. 2011; 63(12):3749-3757. doi: 10.1002/art.30612.

15. Choi HM, Lee YA, Yang HI, Yoo MC, Kim KS. Increased levels of thymosin beta4 in synovial fluid of patients with rheumatoid arthritis: association of thymosin beta 4 with other factors that are involved in inflammation and bone erosion in joints. Int J Rheum Dis. 2011; 14(4):320-324. doi: 10.1111/j.1756-185X.2011.01652.x.

16. Hirota K, Hashimoto M, Yoshitomi H, Tanaka S, Nomura T, Yamaguch T. T cell self-reactivity forms a cytokine milieu for spontaneous development of IL-17+ Th cells that cause autoimmune arthritis. J Exp Med. 2007; 204(1):41-47.

17. Miyake K, Nishida K, Kadota Y, Yamasaki H, Nasu T, Saitou D, et al. Inflammatory cytokine-induced expression of vasohibin-1 by rheumatoid synovial fibroblasts. Acta Med Okayama. 2009; 63(6):349358.

18. van den Berg WB. The role of cytokines and growth factors in cartilage destruction in osteoarthritis and rheumatoid arthritis. Z Rheumatol. 1999; 58(3):136-141.

19. van der Kraan PM. Osteoarthritis year 2012 in review: biology. Osteoarthritis Cartilage. 2012; 20(12): 1447-1450. doi: 10.1016/j. joca.2012.07.010.

20. Abramson S, Krasnokutsky S. Biomarkers in osteoarthritis. Bull NYU Hosp Jt Dis. 2006; 64(1-2):77-81.

21.Patra D, Sandell LJ. Recent advances in biomarkers in osteoarthritis. Curr Opin Rheumatol. 2011; 23(5):465-470. doi: 10.1097/ BOR.0b013e328349a32b.

22. Attur M, Krasnokutsky-Samuels S, Samuels J, Abramson SB. Prognostic biomarkers in osteoarthritis. Curr Opin Rheumatol. 2013; 25(1):136144. doi: 10.1097/BOR.0b013e32835a9381.

23. Naito K, Takahashi M, Kushida K, Suzuki M, Ohishi T, Miura M, et al. Measurement of matrix metalloproteinases (MMPs) and tissue inhibitor of metalloproteinases-1 (TIMP-1) in patients with knee osteoarthritis: comparison with generalized osteoarthritis. Rheumatology (Oxford). 1999; 38(6):510-515.

24. Sandell LJ, Aigner T . Articular cartilage and changes in arthritis. An introduction: cell biology of osteoarthritis. Arthritis Res. 2001; 3(2):107-113.

25. Elmali N, Esenkaya I, Harma A Ertem K, Turkoz Y, Mizrak B. Effect of resveratrol in experimental osteoarthritis in rabbits. Inflamm Res. 2005; 54(4):158-162.

26. Gilston V, Jones HW, Soo CC, Coumbe A, Blades S, Kaltschmidt C et al. NF-kappa B activation in human knee-joint synovial tissue during the early stage of joint inflammation. Biochem Soc Trans. 1997; 25(3):518.

27. Miagkov AV, Kovalenko DV, Brown CE, Didsbury JR, Cogswell JP, Stimpson SA, et al. NF-kappaB activation provides the potential link between inflammation and hyperplasia in the arthritic joint. Proc Natl Acad SciUSA. 1998; 95(23):13859-13864.

28. Meyaard L. The inhibitory collagen receptor LAIR-1 (CD305). J Leukoc Biol. 2008; 83(4):799-803.

29. Martel-Pelletier J, Alaaeddine N, Pelletier JP. Cytokines and their role in the pathophysiology of osteoarthritis. Front Biosci. 1999; 4:D694D703.

30. Ijima Y, Kobayashi M, Kubota E. Role of interleukin-1 in induction of matrix metalloproteinases synthesized by rat temporomandibular joint chondrocytes and disc cells. Eur J Oral Sci. 2001; 109(1):50-59.

31. Smith RJ, Chin JE, Sam LM, Justen JM (1991) Biologic effects of an interleukin-1 receptor antagonist protein on interleukin-1-stimulated cartilage erosion and chondrocyte responsiveness. Arthritis Rheum. 1991; 34(1):78-83. 